\section{Mesa-Redonda: Desigualdades sociais e cobertura vacinal: uso de inquéritos domiciliares}

\section{Comentários}

\section{Elevadas coberturas, equidade e segurança. Desafios do Programa Nacional de imunizações}

\author{
Eliseu Alves Waldman \\ Faculdade de Medicina da Universidade de São Paulo
}

Correspondência: Eliseu Alves Waldman. Faculdade de Saúde Pública, Universidade de São Paulo. Av. Dr. Arnaldo, 715 - 01246-904 São Paulo, SP - Brasil. Tel. (55 11) 3083-5738. Fax (55 11) 3081-2108. E-mail: eawaldma@usp.br
As atividades rotineiras de vacinação, organizadas de forma a atingir elevadas coberturas, iniciam-se no Brasil a partir de 1973 com a implantação do Programa Nacional de Imunizações (PNI), que se destaca entre as experiências mais bem sucedidas da saúde pública em nosso país ${ }^{1,2}$.

Esse sucesso deveu-se, em boa parte, a implementação de medidas que lhe conferiram elevado grau de auto-sustentação. Entre elas: a criação de Comissões Técnicas Assessoras; a formação de equipes especializadas nas três esferas de poder; a implantação e/ou fortalecimento de laboratórios de referência para o controle de qualidade e segurança de imunobiológicos; o desenvolvimento de um ambicioso Programa Nacional de Auto-suficiência de Produtos Imunobiológicos e a criação de um sistema de vigilância para eventos adversos pós-vacina ${ }^{1,2}$.

Além disso, o PNI tem apoiado inquéritos de cobertura vacinal que passam a ser feitos, com certa regularidade, a partir dos anos $80 \mathrm{e}$, mais recentemente, tem incentivado pesquisas focalizando a eficácia/ efetividade e a segurança de vacinas ${ }^{3-6}$.

As atividades de imunização situam-se entre as intervenções de melhor custoefetividade, constituindo um componente obrigatório dos programas de saúde pública $^{7}$. A avaliação da sua efetividade se faz pelo monitoramento da cobertura, eqüidade no acesso e segurança das vacinas ${ }^{8-10}$.

Os sistemas rotineiros de avaliação de cobertura vacinal, por meio de dados administrativos utilizando o número de doses aplicadas, oferecem estimativas pouco precisas, além de não apresentarem sensibilidade suficiente para identificar diferenciais que expressem falta de equidade no acesso.

Por sua vez, é importante que os gestores ao avaliarem o impacto de intervenções em saúde pública na melhora nas condições sanitárias da população, não o façam somente por meio da análise de indicadores globais, mas tentando verificar se os mesmos, de fato, são acompanhados da 
diminuição das desigualdades entre os estratos mais pobres e os mais ricos da população. A inexistência de séries históricas com dados de boa qualidade relativos a indicadores sócio-econômicos e de saúde e a ausência de ao menos duas avaliações seqüenciais em uma mesma comunidade mediante o uso de metodologias comparáveis são obstáculos para tal avaliação ${ }^{11}$.

Moraes aponta em sua apresentação, a relevância de inquéritos periódicos de âmbito nacional com a finalidade de avaliar a cobertura e as desigualdades sociais, mostrando inclusive que esse tipo de estudo pode contribuir para identificar possíveis mudanças na adesão à vacinação em determinados estratos da população, indicando a necessidade de um melhor exame das hipóteses explicativas plausíveis por meio de outras estratégias metodológicas, como a análise qualitativa.

Apresentando os resultados dos inquéritos realizados no município de São Paulo, em 1998 e 2002 3 , Moraes destaca os seguintes pontos: i) ligeiro aumento da cobertura vacinal de 2002 em relação a 1998; ii) coberturas vacinais inferiores às metas do PNI e àquelas obtidas mediante dados administrativos; iii) as crianças de mães alfabetizadas apresentam coberturas mais elevadas do que as analfabetas no estrato social mais rico, porem essa diferença não é observada nos demais estratos socioeconômicos; iv) em 1998, o estrato mais rico e o mais pobre apresentavam as coberturas mais baixas, porem em 2002, houve uma clara tendência de aumento da cobertura nos estratos mais pobres; v) em 2002, verificaram-se expressivos diferenciais de cobertura entre os Distritos Sanitários do município de São Paulo e forte participação da medicina privada na vacinação da população de maior poder aquisitivo (27\%).

Mota*, ao comentar os dados apresentados por Moraes, salienta algumas possíveis limitações da metodologia aplicada. Inicialmente comenta que a técnica de amostragem por conglomerados elabora- da pela OMS, em $1982^{12}$, foi proposta numa época em que as coberturas vacinais eram baixas, o que tornava perfeitamente aceitável, estimativas com intervalos de confiança mais amplos, além disso, recomenda precaução nas estimativas de cobertura vacinal conforme o nível de desagregação/agregação da medida. Assinala, ainda, que por se tratar de uma amostra complexa, é necessário levar em consideração essa característica na análise multivariada.

Em seguida, aborda questões relativas ao registro da condição do vacinado no inquérito, recomendando cuidados especiais na definição de caso (por vacina e por idade), com a possível existência de mais de uma caderneta para cada criança, com a acurácia dos registros e com a definição de critérios que aceitam ou não a vacinação informada.

Por fim, faz menção às medidas de desigualdade social, salientando a importância de aferição da variação na distribuição da renda do chefe da família e da escolaridade da mãe ou responsável, e a importância do fator cor da pele/raça e de sua relação com renda e escolaridade.

Em sua intervenção, Horta** assinala que os inquéritos constituem excelente instrumento para avaliar cobertura, tendência e equidade de acesso a serviços, sendo essenciais na definição de prioridades e na avaliação do impacto das intervenções. Salienta porem, que devem também ser utilizados para identificar barreiras para a vacinação, tanto as relativas aos serviços (oportunidades perdidas, por exemplo), como as individuais, referindose a percepção do risco de eventos adversos. Menciona, além disso, uma limitação dos grandes inquéritos, ao apontar a dificuldade de obtenção de informações relativas a minorias, entre elas, populações indígenas, famílias sem residência fixa e grupos religiosos que não aderem ao uso de tecnologias médicas.

$\mathrm{Na}$ discussão com o plenário, foram igualmente destacados alguns aspectos

* Eduardo Mota Debatedor da Mesa redonda "Desigualdades sociais e cobertura vacinal: uso de inquéritos domiciliares"

** Bernardo Lessa Horta - Debatedor da Mesa redonda "Desigualdades sociais e cober tura vacinal: uso de inquéritos domiciliares" 
como a perda diferencial no estrato de renda mais elevada e a necessidade de estratégias especificas para diminuir a ausência de resposta, que geralmente varia conforme a comunidade estudada e comumente associa-se com o grau de violência nela existente. Em relação à última questão, sugeriu-se como alternativa a realização de inquéritos por fluxo, por meio de entrevistas realizadas na rua.

Mota $^{*}$ assinala em seu comentário final, a necessidade de rediscussão de metas e estratégias dos programas de vacinação levando em consideração o expressivo declínio da morbi-mortalidade por doenças imunopreveníveis, pois possivelmente a mudança desses indicadores foi acompanhada da alteração do risco de infecção pelos agentes envolvidos tanto em termos globais como nos diferentes estratos da população.

Com a finalidade de ampliar os pontos de discussão a respeito deste tema, vale citar a "hipótese da equidade inversa" proposta por Victora e cols ${ }^{11}$ que chama a atenção para o fato de que a incorporação de uma nova intervenção em saúde pública beneficia inicialmente os mais ricos e somente depois os segmentos mais pobres da população, mesmo quando assistimos à melhora substancial dos indicadores médios de saúde na comunidade com a introdução dessa intervenção. Esse aumento da desigualdade pode ser avaliado pela análise da razão desses indicadores entre pobres e ricos, que se reduziria, segundo os autores, quando os mais ricos já tivessem atingido baixos níveis de morbimotalidade.

É interessante notar, nos resultados apresentados por Moraes relativas ao inquérito de $2002^{4}$ que as menores coberturas tenham sido verificadas no estrato mais rico da população e não entre os mais pobres, como seria de se esperar. Para uma análise mais cuidadosa dessa mudança na adesão à vacinação, devemos averiguar se, de fato, esse achado constitui uma tendên- cia. Porém, esse resultado é consistente com o verificado em inquérito realizado em áreas do estado de São Paulo, que aponta o nível de escolaridade como fator inversamente proporcional à adesão à vacinação contra a influenza, entre idosos ${ }^{13}$. Tal fato reforça a sugestão feita por Horta**, de incluir em inquéritos de vacinação questões visando à identificação de barreiras para a vacinação, entre elas a percepção do risco de eventos adversos. A confirmação dessa nova tendência em relação à adesão à vacinação, sugere a possível introdução de um novo fator de desigualdade em saúde, dessa vez associado, não ao acesso aos serviços, mas à informação.

Como salienta Chen ${ }^{8}$, à medida que os programas de vacinação atingem seus objetivos de controlar doenças, verifica-se uma diminuição da percepção de risco em relação a elas e um aumento do temor a eventos adversos pós-vacinação. Por ser um fenômeno identificado em diferentes países, a Organização Mundial de Saúde criou, em 1999, o Global Advisory Committee on Vaccine Safety para assessorá-la em questões relacionadas à segurança de vacinas ${ }^{10,14,15}$.

Finalizando, podemos ainda incluir nesta discussão, a oportunidade de utilizarmos novas tecnologias que juntamente com os inquéritos de cobertura vacinal podem identificar desigualdades. Entre elas, destacamos pela rápida expansão de seu uso em várias regiões do mundo, os registros informatizados de imunização, que tem sua aplicação recomendada para a manutenção de elevadas coberturas vacinais, por meio de sistemas de alerta para incentivar a adesão às vacinações agendadas, atualizar esquemas de vacinação em atraso, aprimorar o monitoramento das coberturas vacinais e identificar áreas de menor cobertura ${ }^{16-18}$.

Tais registros, quando articulados com prontuários eletrônicos, permitem o desenvolvimento de sistemas de vigilância ativa de eventos adversos pós-vacinação,

* Eduardo Mota Debatedor da Mesa redonda "Desigualdades sociais e cobertura vacinal: uso de inquéritos domiciliares"

* Bernardo Lessa Horta - Debatedor da Mesa redonda "Desigualdades sociais e cober tura vacinal: uso de inquéritos domiciliares" 
que além de mais sensíveis do que o sistema passivo utilizado em nosso país, proporciona melhores estimavas do risco desses eventos, uma vez que oferece numeradores e denominadores adequados ${ }^{19}$.

A inclusão, em nossa discussão, do uso de tais registros eletrônicos, se justifica pelo rápido processo de incorporação dessa nova tecnologia no Brasil, onde, em 2007, 62 municípios, dos quais quatro capitais de estado, já o haviam incorporado em sua rede básica de serviços, existindo inclusive um software desenvolvido pelo DATASUS com tal finalidade ${ }^{20}$.

\section{Referências Bibliográficas}

1. Brasil. Programa Nacional de Imunização- 30 anos. Secretaria de Vigilância a Saúde. Ministério da Saúde. 2003.

2. Buss PM, Temporão JG, Carvalheiro JR. Vacinas, Soros \& Imunizações no Brasil. Rio de Janeiro; Editora Fiocruz, 2005.

3. Moraes JC, Barata RB, Ribeiro MCSA, Castro PC. Cobertura vacinal no primeiro ano de vida em quatro cidades do estado de São Paulo, Brasil. Rev Panam de Salud Publ 2000; 8 (5):332-41.

4. Barata RC, Moraes JC, Ribeiro MCSA, Simões O, Castro PC, Mendes JDV, Guibu IA, Gonçalves MJPR. Inquérito de cobertura vacinal no município de São Paulo, 2002. CEALAG;2002.

5. Struchiner CJ, Luz P M, Dourado I, Sato HK, Aguiar SG, Ribeiro JGL, Soares CR, Codeço CT. Risk of fatal adverse events associated with 17DD yellow fever vaccine. Epidemiol Infect 2004; 132: 939-946.

6. Camacho LAB, Aguiar SG, Freire MS, Leal MLF, Nascimento JP, Iguchi T, Lozada JA, Farias RHG and collaborative Group for study of yellow fever vaccine. Reatogenicidade de vacinas contra febre amarela em estudo randomizado, controlado com placebo. Rev Saúde Pública 2005; 39(3):413-20.

7. Begg N, Miller E. Role of epidemiology in vaccine policy. Vaccine 1990; 8:180-89.

8. Chen RT. Evaluation of safety after the events of 11 September 2001: role of cohort and case-control studies. Vaccine 2004; 22:2047-53

9. Delamonica E, Minujin A, Gulaid J. Monitoring equity in immunization coverage. Bull World Health Organ 2005; 83(5):384-91.

10. Duclos F, Delo A, Aguado T, Bilous J, Birminghan M, Kieny MP, Mielstien J, Wood D, Tarantola D. Immunization safety priority project at the World Health Organization. Seminar Pediat Infect Dis 2003; 14(3): 233239.

11. Victora CG, Vaughan JP, Barros FC, Silva AC, Tomasi E. Explaining trends in inequities: evidence from Brazilian child health studies. Lancet 2000;356:1093-8.
12. Henderson RH, Sundaresan T. Cluster sampling to assess immunization coverage: a review of experience with a simplified samplig method. Bull World Health Organ 1982; 60:253-60.

13. Francisco PMSB, Donalisio MR, Barros MBA, César CLG, Carandina L, Goldbaum M. Fatores associados à vacinação contra a influenza em idosos. Pan Am J Public Health 2006;19:259-64.

14. Folb PI, Bernatowska E, Chen R, Clemens J et al. A Global Perspective on Vaccine Safety and Public Health: The Global Advisory Committee on Vaccine Safety. Am Jurnal of Public Health 2004; 94 (11):1926- 1931.

15. Duclos F. A global perspective on vaccine safety. Vaccine 2004; 22:2059-2063.

16. Center for Disease Control and Prevention-CDC. Recommendations of the Advisory Committee on Immunization Practices: Programmatic Strategies to Increase Vaccination Rates - Assessment and Feedback of Provider-Based Vaccination Coverage Information. MMWR 1996; 45(10):219-220.

17. Center for Disease Control and Prevention-CDC. General Recommendations on Immunization: Recommendations of the Advisory Committee on Immunization Practices (ACIP) and the American Academy of Family Physicians (AAFP). MMWR 2002; 51(RR02):1-36

18. Shefer A, Briss P, Rodewald L, Bernier R, Strikas R, Yusuf $\mathrm{H}$ et al. Improving Immunization Coverage Rates: An Evidence-based Review of the Literature. Epidemiol Rev 1999; 21(1):96-142.

19. Zhou W, Pool V, Iskander JK, English_Bullard R, Ball R, Wise RP et al. Surveillance for Safety after Immunization: Vaccine Adverse Event Reporting System (VAERS), USA, 1991-2001. MMWR (Surveillance Summaries) 2003; 52(SS $1): 1-24$

20. Lhum KR. Cobertura vacinal e fatores associados à situação vacinal em Curitiba. [Tese apresentada à Faculdade de Saúde Pública da Universidade de São Paulo; 2008]. 